\title{
475342 - MICROVASCULAR RESPONSE TO CARDIO-PULMONARY BYPASS DURING CARDIAC SURGERY
}

\author{
David Bracco, MD ${ }^{1}$, Marie-Claude Bluteau, BEng ${ }^{1}$, Jean-François Olivier, MD ${ }^{1}$, \\ Dominique Shum Tim, $\mathrm{MD}^{2}$, Christo Tchervenkov, $\mathrm{MD}^{2}$, Thomas Hemmerling, \\ MD $^{1}$
}

1. Anesthesiology, McGill, Montreal, QC, Canada

2. Cardiac Surgery, McGill University, Montreal, QC, Canada

Introduction: Cardiopulmonary bypass (CPB) alters blood flow distribution and organ function. The objective of this study was to investigate the effect of $\mathrm{CPB}$, cooling and rewarming on sublingual microcirculation using Orthogonal Polarization Spectral (OPS) analysis.

Methods: Local REB approval was obtained for this study. This prospective observational study included 20 patients scheduled for cardiac surgery with non-pulsatile CPB. Sublingual microcirculation was determined at seven time points throughout surgery. At each time point, 16 video loops of 5 sec were analyzed. The average and heterogenicity (variance and mean difference to mean) of the vessel diameter distribution, the number of perfused vessels and the type of flow was analyzed. Non-parametric comparisons were used to compare the data.

Results: Baseline microcirculation correlated significantly with the preoperative risk scores. The number of small non-perfused vessels per field correlated positively with the Euroscore predicted mortality $(\mathrm{y}=0,443+10,4 * \mathrm{X}, \mathrm{r} 2=0.45, \mathrm{p}<0.003)$. During CPB, the mean number of small $(8-25 \mu \mathrm{m})$ continuously perfused vessels per field decreased from 11.9 to $9.5(\mathrm{p}<0.05)$. Active or passive cooling and active re-warming did not affect capillary density and flow. With ongoing CPB, there is a microcirculatory adaptation. Discussion: Components of the preoperative risk score have a deleterious effect on baseline microcirculation. The sublingual microcirculation responds to non-pulsatile flow by decreasing the number of small continuously perfused vessels. The fact that microcirculatory flow does not decrease with cooling suggests flow in excess of the metabolic requirements during hypothermia. To assess if these changes are the reason for CPB-related tissular hypoperfusion or an adaptation to non-pulsatile flow requires further studies.

References: This work was supported by the 2006 David S Sheridan Canadian Research Award. 


\section{Microcirclatory changes during cardiac surgery}

A) Nonperfused Vessels [\#field]



C) Sluggishly perfused Vessels [\#field]

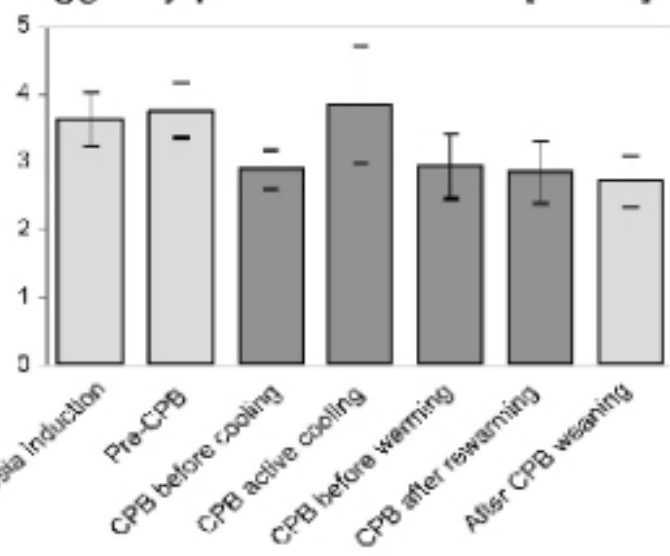

B) Intermittently perfused vessels [\#/field]

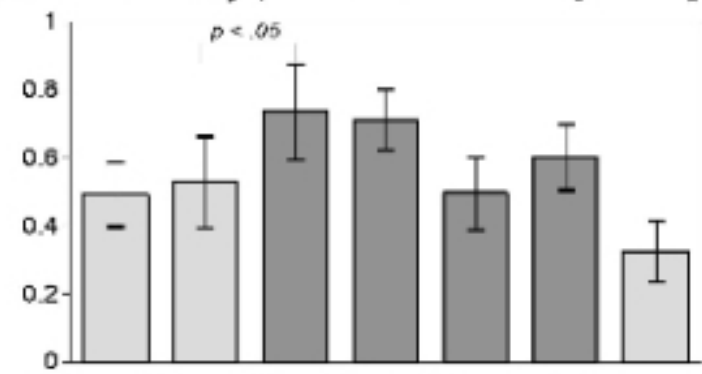

D) Continuously perfused Vessels [\#field]

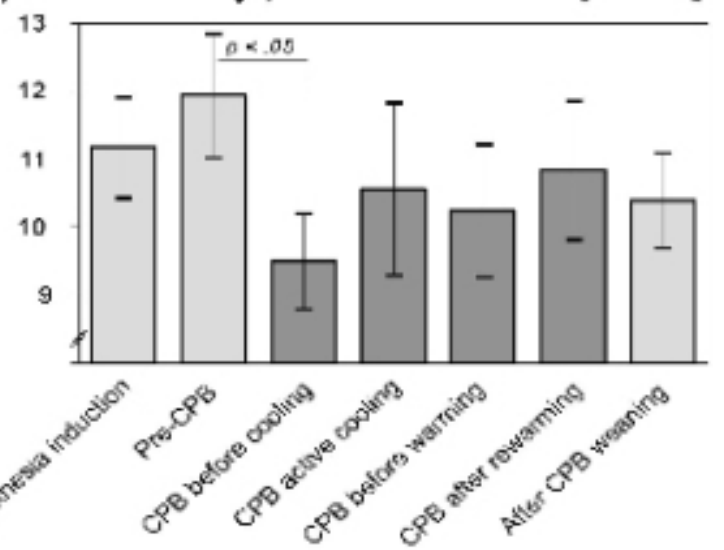

